This leaves us with at least three alternatives: first, that natural population fluctuations are not chaotic and/or are nonstationary; second, that the models are fundamentally flawed; or, third, that environmental forcing needs to be incorporated. Such forcing can easily push a stable map into the chaotic regime ${ }^{7-9}$, and Steele ${ }^{10}$ has suggested that environmental forcing (red noise) superimposed on models containing multiple stable states may lead to the kind of intermittency that could produce a redshift. Whatever the case, Cohen's article poses a dilemma that should stimulate some lively discussion around what remains one of the classic unresolved issues in ecology.

George Sugihara is at the Scripps Institution of Oceanography, University of California, San Diego, 9500 Gilman Drive, La Jolla, California 92093-0202, USA.

\title{
At the roots of nutrition
}

\section{Julian I. Schroeder}

PLANTS grow and develop while remaining rooted in one place. Therefore, identification of the molecular mechanisms through which plants accumulate the often sparse soil macronutrients essential for growth has been a central aim in plant biology. Two papers in this issue add further to our knowledge of such mechanisms. Harrison and van Buuren (page $626^{1}$ ) report the first cloning of a mycorrhizal phosphate-

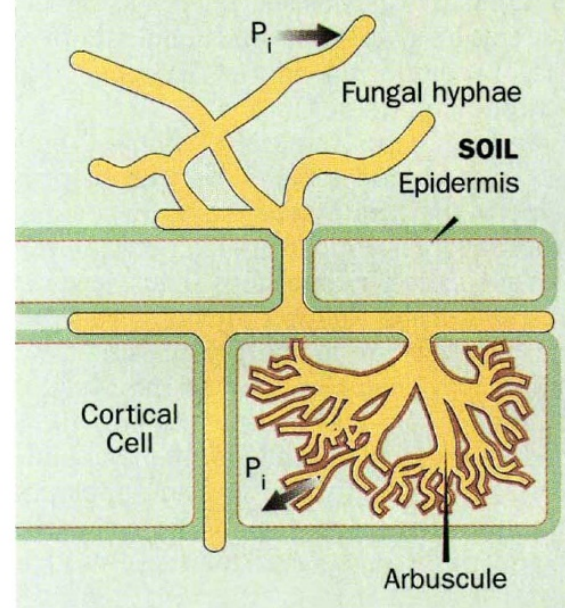

Symbiosis in plant nutrition. A vesicular arbuscular mycorrhizal fungus invades root cortical cells and facilitates phosphate $\left(P_{i}\right)$ uptake from soil.

uptake transporter proposed to function in plant phosphate nutrition; and Tyerman et al. (page $629^{2}$ ) describe the identification of a mechanism for nitrogen accumulation from nitrogen-fixing root nodules.

Macronutrients are usually added to fertilizers, because their limited availability in soils is responsible for reduced plant growth. But some consequent environmental problems, such as large energy use for fertilizer production and groundwater contamination caused by leaching of fertilizers, have added impetus to the quest to identify associated nutrient transporters. A great deal of progress has been made, voltage (patch) clamp and molecular cloning studies having provided first insights into the biophysical uptake mechanisms and molecular structures of key plant transporters for the essential 'NPK' macronutrients (nitrogen, phosphorus and potassium). These include low-affinity ${ }^{3}$ and high-affinity nitrate-uptake transporters ${ }^{4}$ and high-affinity ammonium transporters ${ }^{5}$. Furthermore, low-affinity $\mathrm{K}^{+}$uptake channels ${ }^{6-8}$ and high-affinity $\mathrm{K}^{+}$transporters ${ }^{9,11}$ have been characterized in cell biological, biophysical and molecular terms. But the molecular mechanisms for phosphate uptake have remained unknown.

Harrison and van Buuren' describe the isolation of a complementary DNA encoding a high-affinity phosphate-uptake transporter from vesicular arbuscular (VA) mycorrhizal fungi. The family of VA mycorrhizal fungi form symbiotic associations with most higher plants, including agricultural crops. This association greatly increases plant phosphate uptake (see figure), growth and crop yields ${ }^{11}$, the fungus getting carbon in return. The phosphate transporter, from VA Glomus versiforme mycorrhizae, named GvPT, was cloned by homology to the PHO84 phosphate transporter from yeast ${ }^{12}$. The $G v P T$ sequence encodes a membrane protein, which complements a yeast mutant defective in highaffinity phosphate uptake. GvPT also has a high affinity for phosphate $\left(K_{\mathrm{m}}\right.$ value of about $18 \mu \mathrm{M})$ required for nutrient accumulation. The transporter appears to be expressed predominantly in the external hyphae of the mycorrhizae; VA fungi have extended hyphae that can reach into undepleted soil regions and are crucial for transfer of phosphate and other minerals from the soil to the host plants ${ }^{11}$.

We now have fresh challenges. For example, further research will be needed to characterize the mechanisms of phosphate transfer from mycorrhizae to roots, to identify plant (non-fungal) phosphate uptake transporters, and to determine the effects of transgenic manipulation of $G \nu P T$ on phosphate nutrition. On the practical side, further characterization may allow investigation of the use of $G \nu P T / P H O 84$ homologues for biological removal of leached phosphate.

Nitrogen is a macronutrient that often limits plant growth and crop yields. Leguminous plants have built-in symbiotic factories, root nodules, which contain nitrogen-fixing bacteroids inside organelles (symbiosomes) of the root cells. The bacteroids produce excess ammonia $\left(\mathrm{NH}_{3}\right)$ which accumulates in the symbiosome space. Symbiosomes are surrounded by a specialized organellar membrane, the peribacteroid membrane, but the mechanisms for $\mathrm{NH}_{3}$ or ammonium ion $\left(\mathrm{NH}_{4}{ }^{+}\right)$ uptake across this membrane into the root cytosol have remained unknown.

Tyerman et al..$^{2}$ address this question in a first patch-clamp analysis of the pcribacteroid membrane. They report a prominent, non-selective monovalent cation current in the peribacteroid membrane that is activated by negative potentials on the plant cytosol side of the membrane. As the transporters are most permeable to ammonium, the negative potential would cause $\mathrm{NH}_{4}{ }^{+}$uptake into plants. The cation transporter has a low affinity for $\mathrm{NH}_{4}{ }^{+}$ uptake $\left(K_{\mathrm{m}}\right.$ of about $\left.37.5 \mathrm{mM}\right)$, which correlates with estimated $\mathrm{NH}_{4}{ }^{+}$levels of about $20 \mathrm{mM}$ in the symbiosome space.

Reversal potentials for the $\mathrm{NH}_{4}{ }^{+}$ uptake currents shifted in a strictly Nernstian manner at a symbiosome $\mathrm{NH}_{4}^{+}$concentration of between 3 and $150 \mathrm{mM}$. This shows that the ammonium uptake pathway is passive, which is the thermodynamic definition of a channel. The passive ammonium uptake channels differ from the plant high-affinity $\mathrm{H}^{+}$-coupled $\mathrm{NH}_{4}{ }^{+}$ transporters, which show a $K_{\mathrm{m}}$ of about $10 \mu \mathbf{M}$ (ref. 5).

To approximate the conductance and density of the underlying cation channels, the authors have pursued fluctuation analyses (which can be compared to analysing the noise of uniform raindrops falling on a tent roof to estimate the size and density of drops). This analysis showed that the ammonium channels have unusual, 'non-Lorentzian' properties, pointing to the existence of multiple states, cooperativity among channels or

1. Harrison, M. J. \& van Buuren, M. L. Nature $\mathbf{3 7 8}$ $626-629$ (1995).

. Tyerman, S. D., Whitehead, L. F. \& Day, D. A. Nature 378, 629-632 (1995)

3. Tsay, Y. F., Schroeder, J. I., Feldmann, K. A. \& Crawford N. M. Cell 72, 705-713 (1993).

4. Quesada, A., Galvan, A. \& Fernandez, E. Plant J. 5. $407-419$ (1994).

5. Ninnemann, O., Jauniaux, J. C. \& Frommer, W. B. EMBO J. 13, 3464-3471 (1994)

6. Sentenac, H. et al. Science 256, 663-665 (1992).

7. Anderson, J. A. et al. Proc. natn. Acad. Sci. U.S.A. 89 3736-3740 (1992)

8. Schachtman, D. P. et at. Science 258, 1654-1658 (1992).

9. Schachtman, D. P. \& Schroeder, J. I. Nature $\mathbf{3 7 0}$ 655-658 (1994)

0. Maathuis, F. J. M. \& Sanders, D. Proc. natn. Acad. Sci. U.S.A. 91, 9272-9276 (1994)

11. Smith, S. E. \& Gianinazzi-Pearson, V. A. Rev. PI. Physiol Pl. molcc. Biol. 39, 221-244 (1988).

12. Bun-ya, M., Nishimura, M., Harashima, S. \& Oshima, Y Molec. cell. Biol. 11. 3229-3238 (1991). 
non-typical open-closed transitions. The authors estimate that the single-channel conductance is low, but that channel density in the peribacteroid membrane is high (300-2,400 $\mu^{-2}$ ). Most importantly, they show that total ammonium currents carried by the $\mathrm{NH}_{4}{ }^{+}$-permeable channels can easily accommodate physiological rates of ammonium uptake into the plant. The identification of the $\mathrm{NH}_{4}^{+}$uptake channel in the peribacteroid membrane will allow further characterization of its proposed physiological function and regulation mechanisms (for example, block by calcium $^{2}$ ), and for comparisons with other cation transporters to be made.

The biophysical and cell biological characterization, as well as the cloning, of initial cDNAs encoding key members of the NPK macronutrient transporters are now allowing accurate analyses of numerous physiological questions. For example, interactions of transporters with toxic compounds in soils, such as herbicide uptake by the low-affinity $C H L 1$ nitrate transporter ${ }^{3}$, or the interactions of toxic cations with cation-uptake transporters, are now open to investigation. We are no doubt in for surprises as we find out more about plant macronutrient uptake and the associated environmental stresses.

Julian I. Schroeder is in the Department of Biology and the Center for Molecular Genetics, University of California San Diego, La Jolla, California 92093-0116, USA.

\section{An elusive soluble suppressor}

\section{Anthony S. Fauci}

THE study by Baier et al. on page 563 of this issue ${ }^{1}$ describes the identification of a soluble factor (lymphokine) secreted by $\mathrm{CD}^{+}$-bearing $\mathrm{T}$ lymphocytes that inhibits the replication of the human immunodeficiency virus (HIV). The potential implications of this study are substantial.

A variety of classical antibody and cellbased immune responses have been described that contribute to anti-HIV immunity, including neutralizing antibodies, antibody-dependent cellular cytotoxicity, natural killer cells, and HIV-specific cytotoxic $\mathrm{T}$ cells (reviewed in ref. 2 ). Several years ago, a phenomenon of noncytolytic suppression of HIV replication was described ${ }^{3}$ : the suppression was shown to be due to a soluble factor or factors derived from $\mathrm{CD} 8^{+} \mathrm{T}$ lymphocytes of HIV-infected individuals, and these findings were subsequently corroborated by several laboratories (reviewed in ref. 4). Similar phenomena and factors have been described in the simian immunodeficiency virus (SIV) model of HIV infection in the monkey ${ }^{5}$ (also reviewed in ref. 4).

Despite continued attempts to isolate the factor(s), however, their precise identification has remained elusive. It is likely that the difficulties can be explained at least in part by the facts that there may be a family of suppressor factors, and that attempts to purify and assay just one of them have resulted in marked decreases and inconsistencies in suppressor activity compared to that of the crude supernatants. This may very well be the case with the human factor cloned by Baier and colleagues, which gives lower levels of suppression than those seen in studies on crude supernatants ${ }^{3-5}$.

Baier et al. identified a factor that had been previously described ${ }^{6}$ in a different context from HIV. The factor has been designated lymphocyte chemoattractant factor (LCF) or IL-16, and it suppresses HIV replication in $\mathrm{CD}^{+}$T-lymphocytedepleted human peripheral blood mononuclear cells ${ }^{1}$. The current authors had previously identified an "immunodeficiency-virus-suppressing lymphokine" (ISL) that was secreted by $\mathrm{CD} 8^{+} \mathrm{T}$ cells of African green monkeys (AGM) and which potently suppressed the replication of SIV in monkey cells as well as the replication of HIV in human $\mathrm{CD}^{+} \mathrm{T}$ lymphocytes ${ }^{5}$. Baier et al. note that the sequences encoding human IL-16 and AGM IL-16 are very alike, in that the IL-16 genes of humans differ from those of African green monkeys in only 16 of their 390 coding nucleotides, resulting in only seven nonclustered amino-acid changes.

It is not surprising, despite the intensive and hitherto unsuccessful search for the identity of the soluble suppressor factors(s) of HIV replication, that at least one of the factors is a lymphokine with other wellknown functions apart from suppression of HIV replication. In this regard, it is highly likely that other discrete factors with different non-HIV related functions will be described that also suppress the replication of HIV. This might lead to understandable confusion as to the true identity of the suppressor factor; in fact, it is likely that a combination of factors will be required for optimal suppression.

It is out of the question that soluble factor(s) have emerged in the host species specifically to suppress HIV replication. Rather, it is conceivable that the virus has evolved to use existing factors of its host to its own advantage. Because the suppression of virus replication by the newly cloned factor is non-cytolytic (that is, it does not involve host destruction of infected host cells), a consequence might be the establishment of latency, with the resulting implication of prolonged viral infection without killing the host.

Although $\mathrm{CD}^{+}$T-lymphocyte-derived LCF (IL-16) is a natural CD4 ligand ${ }^{7}$, the ways in which it inhibits HIV replication are unknown. It is likely that those mechanisms will be quickly elucidated as investigators come to grips with the cloned factor. It will be important to determine the effect of the factor on replication of primary isolates of HIV, as well as on in vitro endogenous replication of virus in cells from HIV-infected individuals.

Work on the $\mathrm{CD}^{+}$T-lymphocyte-suppressor phenomenon has been seriously impeded by the unavailability of purified factors, necessitating the use of crude or only partially purified supernatants. In essence, the new study ${ }^{1}$ opens up fresh avenues of research into pathogenesis, treatment and vaccine development. Of particular importance is the possibility of a novel form of 'immune-based' therapy. Transplantation of tissue and reinfusion of ex vivo expanded autologous immunecompetent cells into HIV-infected individuals have met with the difficulties common to any such approach, including tissue rejection, graft-versus-host reactions and unforeseen toxicities (reviewed in ref. 8). The availability of a soluble factor with demonstrable ability to suppress HIV replication will certainly expand the options for experimental therapy. It is particularly relevant that there is considerable homology between human IL-16 and AGM IL-16, adding importance to the SIV monkey model of HIV infection.

Of course, there is no guarantee that such therapy would be effective, and more than a decade of experience with HIV has taught us to be conservative in our projections of success until properly designed and controlled clinical trials have been performed. However, at the very least, the availability of this and other HIV-suppressive factors alone or in combination will tell us a great deal about HIV pathogenesis, and in this respect will provide the framework for the development of strategies for therapy and vaccine development.

Anthony S. Fauci is at the National Institute of Allergy and Infectious Diseases, National Institutes of Health, Bethesda, Maryland 20892, USA.

\footnotetext{
1. Baier, M., Werner, A., Bannert, N., Metzner, K. \& Kurth, R. Nature 378, 563 (1995).

Pantaleo, G., Graziosi, C. \& Fauci, A. S. New Engl. J. Med. 328, 327-335 (1993)

3. Walker, C. M. et al. Science 234, 1563-1566 (1986).

4. Mackewicz, C. \& Levy, J. A. AIDS Res. Hum. Retrovir. 8 , 1039-1050 (1992).

5. Ennen, J. et al. Proc. natn. Acad. Sci. U.S.A. 91 7207-7211 (1994).

6. Cruikshank, W. W. et al. Proc. natn. Acad. Sci. U.S.A. 91, 5109-5113 (1994)

7. LaBerge, S. et al. J. Immun. 155, 2902-2910 (1995).

8. Fauci, A. S. Science 262, 1011-1018 (1993)
} 